\title{
QUALITÉ NUTRITIVE DE L'ALIMENTATION NATURELLE DU TILAPIA OREOCHROMIS NILOTICUS EN ÉLEVAGE EXTENSIF DANS DES ÉTANGS DE BARRAGE (CÔTE D'IVOIRE)
}

\author{
M.C. BLE (1), R. ARFI (2), A.F. YEBOUA (3), K.J. DIOPOH (3)
}

(1) Centre de Recherches Océanologiques, Département Aquaculture, BP V 18 Abidjan, Côte d'Ivoire. Courriel : blecel@hotmail.com

(2) Institut de Recherche pour le Développement, Centre d'Océanographie, Station Marine d'Endoume, rue de la batterie des lions, 13007 Marseille Courriel : robert.arfi@univmed.fr

(3) Université de Cocody Abidjan, UFR de Biosciences, 01 BP V 34 Abidjan 01, Côte d'Ivoire.

Reçu le 7 novembre 2005

Accepté le 19 mars 2007

Received November 7, 2005

Accepted March 19, 2007

\section{RÉSUMÉ}

La composition chimique des sources alimentaires (périphyton, matières en suspension et sédiment) et celle des contenus stomacaux d'Oreochromis niloticus en élevage extensif dans deux barrages situés en zone rurale (Gueyo, Sud-Ouest de la Côte d'Ivoire) ont été déterminées afin d'apprécier la qualité de la nourriture naturelle ingérée par ces poissons.

Les proportions des différents constituants chimiques mesurés dans ces trois sources alimentaires laissent apparaître la part importante que représentent les matières organiques hydrolysables dans le périphyton $(65$ et $67 \%$ aux barrages 1 et 2, respectivement), alors que les matières minérales constituent plus de la moitié du poids sec total des matières en suspension (59 et $63 \%$ ) et de la ressource sédimentaire (76 et $63 \%)$. Sur les deux sites, les fibres représentent moins de $15 \%$ de ces trois ressources. Les teneurs en protéines (19\%) et le ratio Protéines/Énergie $\left(17 \mathrm{mg}^{\mathrm{k}} \mathrm{kJ}^{-1}\right)$ observés dans la ressource périphytique sont respectivement 2 et 3 fois supérieurs aux valeurs mesurées dans les deux autres ressources.

L'analyse biochimique des contenus stomacaux montre que les protéines sont présentes en très faibles proportions dans la nourriture ingérée par $O$. niloticus ( 7 et $11 \%$ dans les deux barrages). Par contre, les proportions en fibres (12 et $16 \%$ ) et lipides (11 et $8 \%$ ) de même que les ratios Protéines/Énergie (6 et $12 \mathrm{mg}^{\mathrm{k}} \mathrm{kJ}^{-1}$ ) caractérisant l'alimentation semblent répondre aux exigences de croissance chez cette espèce de tilapia. Les poids moyens des poissons en fin de cycle d'élevage traduisent la capacité d'O. niloticus à présenter une croissance correcte à partir d'une alimentation pauvre en matières azotées pourvu qu'une faible densité soit appliquée dans les sites d'élevage.

Mots-clés: Oreochromis niloticus, barrages piscicoles, alimentation naturelle, qualité nutritive. 


\section{NUTRITIVE VALUE OF NATURAL FOOD OF TILAPIA OREOCHROMIS NILOTICUS IN EXTENSIVE AQUACULTURE RESERVOIRS (IVORY COAST)}

\section{ABSTRACT}

The chemical composition of natural food sources (periphyton, suspended solids and sediment) and gut content of Oreochromis niloticus in two extensive aquaculture reservoirs from a rural area (Gueyo, southwest of Ivory Coast) were determined in order to assess the quality of the natural food ingested.

The percentage of the chemical components in the three food sources in the two sites show an important part of hydrolysable organic matter in the periphyton (65 and $67 \%$ ), even though the mineral matter represents more than half of the total dry weight of suspended solids (59 and 63\%) and sediment (76 and 63\%). In the two sites, fibers represent less than $15 \%$ of these three resources. Protein contents (19\%) and Proteins/ Energy ratio $\left(17 \mathrm{mg}^{\mathrm{kJ}} \mathrm{J}^{-1}\right)$ in periphyton are respectively 2 and 3 times above the values measured for the two other resources.

The biochemical analysis of the gut contents shows that proteins are in low percentage in the food ingested by $O$. niloticus ( 7 and $11 \%$ in the two sites). On the other hand, the percentages of fibers (12 and 16\%) and lipids (11 and $8 \%$ ) as well as the Proteins/Energy ratios $\left(6\right.$ and $\left.12 \mathrm{mg} \cdot \mathrm{kJ}^{-1}\right)$ seem to meet the growth requirements of the studied species. The average weights of the fishes at the end of the farming cycle are indicative of the ability of $O$. niloticus to grow on a poor food context characterized by low nitrogen compounds, as long as a low density is applied in the aquaculture sites.

Key-words: Oreochromis niloticus, aquaculture reservoirs, natural food, nutritive quality.

\section{INTRODUCTION}

En Côte d'Ivoire, du fait de la présence de nombreux bas-fonds et de cours d'eau, on assiste depuis quelques années à l'émergence d'une pisciculture de type artisanal dans les régions du Centre-Ouest et du Sud-Ouest. Toutefois, la production piscicole demeure faible, surtout dans les zones rurales où l'accès aux intrants est limité. De plus, la plupart des intrants dont disposent les pisciculteurs ont une faible valeur nutritive (MORISSENS et al., 1996). C'est pourquoi des modèles piscicoles extensifs favorisant la productivité naturelle du milieu et son exploitation directe par le poisson sont préconisés. Le principe consiste à mettre à la disposition des poissons de grandes surfaces de retenues d'eau appelées "barrages » afin qu'ils trouvent suffisamment de nourriture naturelle. Bien que l'importance des ressources végétales dans l'alimentation de la plupart des espèces piscicoles et plus spécifiquement des tilapias ait été mise en évidence (LAUZANNE, 1978 ; SCHROEDER, 1978 ; DEMPSTER et al., 1993), il est également montré que cette source de nourriture naturelle est de qualité variable et souvent mauvaise (BOWEN, 1976, 1979, 1987 ; EYUALEM et GETACHEW, 1992). Ceci pourrait constituer un obstacle au succès des consommateurs primaires, malgré la grande abondance de ces ressources nutritives naturelles (BOWEN, 1988). Cette étude a pour objectif de préciser l'environnement nutritionnel du tilapia Oreochromis niloticus en élevage extensif dans les barrages, en mettant en évidence les caractéristiques nutritives de ces sources alimentaires afin d'améliorer le fonctionnement de ces systèmes extensifs de production en recommandant l'utilisation d'intrants et/ou de dispositifs susceptibles de promouvoir le développement d'une ressource trophique favorable. 


\section{MATÉRIEL ET MÉTHODES}

\section{Caractéristiques et environnement des barrages piscicoles}

Les barrages étudiés sont des retenues d'eau situées dans deux bas-fonds différents et appartenant à un même pisciculteur. Le barrage 1, qui connaît son premier cycle d'élevage, a une surface de $900 \mathrm{~m}^{2}$. Le barrage 2, beaucoup plus grand, s'étend sur $10000 \mathrm{~m}^{2}$ et a déjà été empoissonné. Ces deux sites sont choisis du fait de leurs performances techniques. Ils comportent chacun un moine permettant de réguler le niveau d'eau et d'effectuer la vidange en fin de cycle de production. Ces barrages sont régulièrement suivis par le pisciculteur qui contrôle tous les événements qui surviennent sur les sites. Par ailleurs, le niveau d'eau lors de l'étude était suffisant pour effectuer l'empoissonnement. A proximité des moines, la profondeur était de 1,2 $\mathrm{m}$ et 1,5 $\mathrm{m}$ dans les barrages 1 et 2 respectivement, avec une transparence de l'eau comparable, la profondeur de disparition du disque de Secchi étant de 0,4 m pour les deux sites. Mal déboisés lors de leur mise en eau, ces barrages contiennent de nombreux morceaux de bois morts. Les pisciculteurs pratiquent de façon générale la polyculture, en élevant plusieurs espèces présentant des régimes alimentaires différents (Oreochromis niloticus, Heterotis niloticus, Heterobranchus isopterus) dans un même milieu. Cette technique permet d'accroître le revenu final des pisciculteurs, sans augmenter significativement l'investissement initial. L'espèce principale d'élevage et qui fait l'objet de notre étude est le tilapia O. niloticus.

L'empoissonnement est réalisé par le pisciculteur, qui fixe également la durée du cycle d'élevage en fonction de ses moyens et de sa disponibilité. Cette option a été retenue afin d'apprécier les réalités de la pratique piscicole en zone rurale. Le barrage 1 est empoissonné avec 227 juvéniles mâles d'O. niloticus (soit 0,3 poisson.m ${ }^{-2}$ ) d'un poids initial moyen de $61,6 \pm 8,5 \mathrm{~g}$ et avec 20 Hemichromis fasciatus (soit un Hemichromis pour 10 tilapias). Les Hemichromis sont des poissons carnivores permettant de contrôler la population initiale des tilapias, en éliminant d'éventuels alevins qui pourraient être présents du fait d'un mauvais sexage lors de l'empoissonnement. La durée du cycle d'élevage est de 187 jours. Dans le second barrage, l'empoissonnement est réalisé avec 792 alevins mâles d'O. niloticus (soit 0,1 poisson. $\mathrm{m}^{-2}$ ) d'un poids moyen de 13,4 $\pm 2,1 \mathrm{~g}$ et avec $20 \mathrm{H}$. fasciatus. La durée du cycle de production est de 223 jours.

\section{Stratégie d'échantillonnage}

Les échantillonnages au sein des deux barrages ont lieu en fin de cycle d'élevage. Pour ne pas être perturbé par la pêche (vidange des barrages et récolte des poissons) qui s'effectue tôt le matin, l'échantillonnage est réalisé la veille. Les prélèvements portent sur les sources alimentaires du milieu et les contenus stomacaux et intestinaux d'Oreochromis niloticus. L'échantillonnage des sources alimentaires potentielles a porté sur le périphyton, les matières particulaires en suspension et le matériel sédimenté qui représentent les compartiments nutritifs susceptibles d'être exploités par O. niloticus, espèce microphytophage à tendance omnivore.

Le périphyton est prélevé sur la surface de 4 fagots de bois prélevés dans l'eau. Sur la partie du bois recouverte de périphyton, une surface est délimitée. Le périphyton est alors gratté à l'aide d'un couteau et l'échantillon est placé dans des tubes contenant de l'eau du milieu filtrée, à laquelle on ajoute quelques gouttes de formol (concentration finale $\sim 5 \%$ ). La circonférence du substrat et la hauteur de la partie grattée sont ensuite mesurées pour calculer la surface échantillonnée.

La technique de mesure des matières en suspension (MES) consiste à prélever un grand volume d'eau (60 litres) à différents endroits et profondeurs (surface, 0,5 et $1 \mathrm{~m}$ ) du barrage au moyen d'une bouteille fixée à une perche. La bouteille fermée est plongée 
lentement dans l'eau jusqu'à la profondeur de prélèvement et son ouverture s'effectue au niveau souhaité en tirant sur une corde reliant le bouchon de fermeture et la perche. Après ajout de quelques gouttes de formol à $35 \%$, puis décantation pendant 24 heures et élimination du surnageant à l'aide d'un tuyau terminé par un tube en $T$, le matériel est recueilli dans des tubes pour être séché à l'étuve à $105^{\circ} \mathrm{C}$.

Le prélèvement de sédiment combinant le film des matières en suspension ayant sédimenté et la fraction minérale est effectué par carottage, à l'aide d'un tube translucide de $1,9 \mathrm{~cm}$ de diamètre que l'on enfonce délicatement dans le sédiment. La carotte étant légèrement inclinée, on ferme l'extrémité supérieure à l'aide d'un bouchon. Puis, à l'aide d'un autre bouchon, on ferme l'autre extrémité de la carotte (en évitant les infiltrations d'eau qui pourraient perturber l'échantillon) et l'ensemble est retiré de l'eau. L'épaisseur du sédiment prélevé est de $3 \mathrm{~cm}$. Les dix points de prélèvement sont répartis de manière à couvrir toute l'étendue du barrage.

Les poissons sont pêchés à partir de 15 heures à l'aide d'une senne de $14 \mathrm{~mm}$ de vide de maille dans une zone de pêche délimitée dans le barrage. Ce procédé de pêche à la senne a l'avantage de minimiser la digestion post-capture (WINDELL et BOWEN, 1978). Après avoir récupéré immédiatement dix poissons au hasard, leur taille et leur poids sont déterminés et les poissons disséqués pour prélever leur estomac et les dix derniers centimètres de leur intestin. Ces échantillons sont immédiatement conservés dans du formol $10 \%$ (BOWEN, 1983) et les contenus stomacaux et intestinaux (fèces) sont par la suite séparés de leurs enveloppes respectives.

Les paramètres de croissance et de production en fin d'élevage sont suivis tous les mois et sont estimés à partir du taux de survie, du gain moyen quotidien et du rendement de croissance.

\section{Analyses}

Au laboratoire, on procède aux analyses chimiques et biochimiques des échantillons (périphyton, MES, sédiment, contenus stomacaux et fèces), préalablement séchés à l'étuve à $105^{\circ} \mathrm{C}$ jusqu'à obtention d'un poids constant. Ces échantillons sont ensuite broyés pour les différentes analyses qui portent sur la détermination de leur contenu en matières organiques (poids sec sans cendre), en fibres, en protéines et en lipides et sur leur valeur énergétique.

- Les constituants chimiques du matériel prélevé sont déterminés suivant la méthode de BUDDINGTON (1980). Un échantillon de $30 \mathrm{mg}$ environ (A) est hydrolysé dans un tube avec de l'acide acétique glacial ( $80 \%)$ et de l'acide nitrique concentré. Le mélange est porté à ébullition à $125^{\circ} \mathrm{C}$ dans un bloc chauffant pendant 40 minutes. Après refroidissement, les échantillons sont récupérés sur des filtres en fibres de verre (filtres Whatman GF/C, porosité 1,2 $\mu \mathrm{m}$ ), préalablement calcinés et pesés (B). L'ensemble est successivement rincé avec du toluène, du diéthyl-éther et de l'éthanol (70 \%). Les filtres sont ensuite séchés à l'étuve à $105^{\circ} \mathrm{C}$ pendant 30 minutes, pesés (C), calcinés au four à $550^{\circ} \mathrm{C}$ pendant une demi-heure et pesés à nouveau (D). Les teneurs en $\mathrm{MO}$ (Matière organique totale), $\mathrm{MOH}$ (Matière organique hydrolysable), MORH (Matière organique résistante à l'hydrolyse) et en Min (Minéraux) sont déduites de ces différentes étapes :

$$
\begin{array}{ll}
\% \mathrm{MO}=\frac{(\mathrm{A}+\mathrm{B}-\mathrm{D})}{\mathrm{A}} & \% \mathrm{Min}=\frac{(\mathrm{D}-\mathrm{B})}{\mathrm{A}} \\
\% \mathrm{MORH}=\frac{(\mathrm{C}-\mathrm{D})}{\mathrm{A}} & \% \mathrm{MOH}=\% \mathrm{MO}-\% \mathrm{MORH}
\end{array}
$$


- Les lipides sont extraits au moyen de diéthyl-éther dans un soxhlet pendant 3 heures à partir d'un échantillon de poids connu (environ $30 \mathrm{mg}$ ) placé dans du papier filtre Whatman (GF/C) de poids connu également. A la fin de l'extraction, l'ensemble (papier filtre et échantillon) est laissé à l'air libre pendant 10 à 15 minutes, puis séché à l'étuve à $105^{\circ} \mathrm{C}$ pendant 30 minutes pour être ensuite refroidi au dessiccateur et pesé. La quantité de lipides est estimée par différence de poids avant et après l'extraction (GOLTERMAN et al., 1978).

- Le dosage des protéines totales se fait suivant la méthode utilisée par ALLEN (1981). Celle-ci consiste à réaliser une hydrolyse alcaline d'un échantillon d'environ $12 \mathrm{mg}$ avec de la soude $10 \mathrm{~N}$ dans un autoclave (Lequeux, 91410 Dourdan) pendant 20 minutes à $121^{\circ} \mathrm{C}$. Cette base est ensuite neutralisée par l'acide acétique glacial. Une fraction des échantillons est diluée et on y ajoute le réactif à la ninhydrine, puis l'ensemble est placé dans un bloc chauffant à $105^{\circ} \mathrm{C}$ pendant 20 minutes. La même opération est effectuée en parallèle sur une gamme étalon réalisée à partir d'une solution d'albumine sérique de bœuf $\left(400 \mu \mathrm{g} \mathrm{l}^{-1}\right)$. La lecture de la densité optique est effectuée au spectrophotomètre (Beckman DU-64) à $570 \mathrm{~nm}$ après ajout de n-propanol (50\%). Les données sont converties en quantités d'acides aminés totaux à l'aide d'une courbe d'étalonnage et de l'équation de la droite de la régression linéaire qui en résulte.

- La quantité d'énergie est déterminée à l'aide d'une bombe calorimétrique à partir de $10 \mathrm{mg}$ d'échantillon.

- Le coefficient d'utilisation digestive apparente (CUDa) est calculé pour les différents composés (matière organique hydrolysable et protéines) suivant la méthode de CONOVER (1966), en utilisant les minéraux comme matériel de référence :

$$
\begin{aligned}
& C U D a_{M O H}=100 \times \frac{\left(\frac{M O H_{c s}}{\operatorname{Min}_{C s}}-\frac{M O H_{f}}{\operatorname{Min}_{f}}\right)}{\left(\frac{M O H_{c s}}{\operatorname{Min}_{c s}}\right)} \\
& C U D a_{\text {proteines }}=100 \times \frac{\left(\frac{\text { protéines }_{\mathrm{cs}}}{\operatorname{Min}_{\mathrm{cs}}}-\frac{\text { protéines }_{f}}{\operatorname{Min} f}\right)}{\left(\frac{\text { protéines }_{c s}}{\operatorname{Min}_{c s}}\right)}
\end{aligned}
$$

Avec :

$\mathrm{MOH}_{\mathrm{cs}}, \mathrm{Min}_{\mathrm{cs}}$ et Protéines $_{\mathrm{cs}}$ : teneurs en matière organique hydrolysable, en minéraux et en protéines des contenus stomacaux, respectivement.

$\mathrm{MOH}_{\mathrm{f}}, \mathrm{Min}_{\mathrm{f}}$ et Protéines $\mathrm{f}$ : teneurs en matière organique hydrolysable, en minéraux et en protéines des fèces, respectivement.

La digestibilité, le rapport protéines/énergie $(P / E)$ et les paramètres chimiques et biochimiques ainsi déterminés sont utilisés comme indicateurs d'appréciation de la valeur nutritive de l'alimentation des poissons. 


\section{RÉSULTATS ET DISCUSSION}

\section{Potentialités nutritives des sources alimentaires}

La qualité des apports nutritifs joue un rôle important dans le comportement et la stratégie alimentaire des poissons (BOWEN et al., 1995). Ce caractère mis en évidence à travers l'analyse des indicateurs chimiques et biochimiques de la valeur nutritive, est variablement apprécié au niveau des différentes sources alimentaires des barrages.

Les teneurs en composés organiques de la ressource périphytique issue des deux sites indiquent un pourcentage élevé de matières organiques hydrolysables (65\%) (Figure 1a). Cette fraction "digestible » représente 80 à $85 \%$ du matériel organique total de la ressource disponible dans les deux barrages, ce qui constitue une part importante des sources de nourriture exploitable par les poissons, bien que les quantités de matières organiques non hydrolysables (164 mg. $\mathrm{g}^{-1}$ de poids sec) soient plus élevées dans le périphyton du barrage 2. Les proportions du matériel inorganique $\left(236 \mathrm{mg} \cdot \mathrm{g}^{-1}\right.$ de poids sec) sont par contre plus importantes dans la ressource périphytique du barrage 1. Ces taux apparaissent toutefois élevés dans le matériel des deux sites, et cela semblent être beaucoup plus le fait de particules minérales piégées au sein de la biomasse périphytique, comme il a été constaté dans d'autres études (BLÉ et al., 2006). Dans l'ensemble, les biomasses de périphyton ainsi que les caractéristiques chimiques ne sont pas significativement différentes entre le périphyton des deux barrages (Tableau I). Les composantes organiques et minérales présentent des valeurs comparables à celles mesurées dans le périphyton se développant sur les bambous des acadjas, une structure d'élevage extensif expérimentée en lagune Ébrié (BLÉ et al., 2006). La teneur protéinique ( $19 \%$ en moyenne de la matière sèche) et le rapport Protéines/Énergie (18 mg.kJ $\left.{ }^{-1}\right)$ sont également proches des résultats rapportés par AZIM et al. (2003) qui notent $17 \%$ de protéines et un ratio $\mathrm{P} / \mathrm{E}$ de $20 \mathrm{mg} \cdot \mathrm{kJ}^{-1}$ dans le périphyton colonisant des substrats artificiels testés en étang. Le contenu énergétique (9 à $13 \mathrm{~kJ} \cdot \mathrm{g}^{-1}$ de poids sec) et les teneurs en protéines diffèrent par contre des valeurs obtenues au niveau du matériel périphytique prélevé sur des substrats analogues aux morceaux de bois issus des barrages (AZIM et al., 2002a). Ces différences d'ordre quantitative et qualitative seraient liées à la composition taxonomique du peuplement périphytique et à la nature des substrats (GUIRAL et al., 1993 ; KESHAVANATH et al., 2001 ; AZIM et al., 2002b). Bien que présentant une qualité nutritive variable, la ressource périphytique semble constituer la source de nourriture préférentielle des poissons planctophages lorsque ces derniers ont à opérer un choix trophique (DEMPSTER et al., 1993). Les études menées par ces auteurs chez des juvéniles d'Oreochromis niloticus ont en effet montré que l'alimentation par broutage et raclage sur substrats (donc du périphyton) était plus efficace que l'alimentation par filtration. La qualité nutritive de cette source alimentaire ainsi que l'effet positif des substrats sur la productivité d'O. niloticus ont été établis (HUCHETTE et al., 2000 ; AZIM et al., 2003). Les nombreuses traces de broutage laissées par les poissons sur les morceaux de bois des barrages semblent confirmer l'intérêt des tilapias pour cette ressource.

Les matières en suspension (MES) formant la deuxième ressource trophique étudiée dans les barrages regroupent des particules organiques (organismes planctoniques, assemblages de bactéries et de détritus) et minérales présentes dans la colonne d'eau. Leurs quantités sont plus importantes pour le barrage $2\left(11 \mathrm{mg}^{-1}\right)$ et cette différence avec la ressource du barrage $1\left(7 \mathrm{mg.l}^{-1}\right)$ résulte essentiellement du taux élevé du matériel minéral (Tableau I). A l'inverse du périphyton, ces MES sont largement constituées de composés minéraux qui forment, avec la fraction organique résistante à l'hydrolyse, 66 à $74 \%$ de la ressource en constituants non digestibles (Figure 1b). Ces taux élevés de composés inorganiques proviendraient en majeure partie des eaux de ruissellement fortement chargées en argiles. La morphologie et la structure des barrages (semi-ouverts, faible profondeur, bas-fonds fermés) faciliteraient le transit des eaux de ruissellement. De 


\section{Tableau I}

Caractéristiques chimiques et biochimiques déterminées dans le périphyton, les MES, le sédiment et les contenus stomacaux d'O. niloticus issus des deux barrages. Comparaison des moyennes à l'aide d'un test non paramétrique de Mann-Whitney (ns : non significatif).

\section{Table I}

Chemical and biochemical characteristics measured in periphyton, sediment, suspended solids and gut contents of 0 . niloticus in the two reservoirs. Differences between groups are tested using Mann-Whitney rank sum test (ns: non significant).

\begin{tabular}{|c|c|c|c|c|c|c|c|c|}
\hline & \multirow{2}{*}{$\begin{array}{l}\text { Caractéristiques } \\
\text { nutritionnelles }\end{array}$} & \multicolumn{3}{|c|}{ Barrage 1} & \multicolumn{3}{|c|}{ Barrage 2} & \multirow[b]{2}{*}{$\begin{array}{l}\text { Diffé- } \\
\text { rences }\end{array}$} \\
\hline & & Moyenne & $\begin{array}{c}\text { Écart- } \\
\text { type }\end{array}$ & $\mathbf{n}$ & Moyenne & $\begin{array}{c}\text { Écart- } \\
\text { type }\end{array}$ & $\mathbf{n}$ & \\
\hline \multirow{9}{*}{ Périphyton } & Poids sec total $\left(\mathrm{g} \cdot \mathrm{m}^{-2}\right)$ & 28,5 & 6,3 & 4 & 21,8 & 4,8 & 3 & ns \\
\hline & $\mathrm{MOH}\left(\mathrm{g} \cdot \mathrm{m}^{-2}\right)$ & 18,4 & 4,2 & 4 & 14,5 & 2,7 & 3 & ns \\
\hline & $\mathrm{MORH}\left(\mathrm{g} \cdot \mathrm{m}^{-2}\right)$ & 3,4 & 1,1 & 4 & 3,7 & 1,5 & 3 & ns \\
\hline & Minéraux $\left(\mathrm{g} \cdot \mathrm{m}^{-2}\right)$ & 6,7 & 1,0 & 4 & 3,6 & 0,5 & 3 & ns \\
\hline & Protéines (mg.g ${ }^{-1}$ poids sec) & 180,2 & 46,1 & 4 & 201,3 & 57,2 & 3 & ns \\
\hline & Protéines $\left(\mathrm{mg} \cdot \mathrm{g}^{-1} \mathrm{MO}\right)$ & 257,6 & 90,4 & 4 & 270,4 & 102,9 & 3 & ns \\
\hline & Énergie (kJ.g ${ }^{-1}$ poids sec) & 9,4 & 1,8 & 4 & 12,5 & 3,1 & 3 & ns \\
\hline & Énergie (kJ.g $\left.\mathrm{g}^{-1} \mathrm{MO}\right)$ & 14,2 & 2,3 & 4 & 15,8 & 4,6 & 3 & ns \\
\hline & $\mathrm{P} / \mathrm{E}\left(\mathrm{mg} \cdot \mathrm{kJ}^{-1}\right)$ & 18,1 & 5,9 & 4 & 17,1 & 4,0 & 3 & ns \\
\hline \multirow{9}{*}{ MES } & Poids sec total $\left(\mathrm{mg} . \mathrm{l}^{-1}\right)$ & 6,9 & - & 1 & 11,2 & - & 1 & - \\
\hline & $\mathrm{MOH}\left(\mathrm{mg} . \mathrm{I}^{-1}\right)$ & 2,3 & - & 1 & 3,1 & - & 1 & - \\
\hline & $\mathrm{MORH}\left(\mathrm{mg} . \mathrm{l}^{-1}\right)$ & 0,5 & - & 1 & 1,0 & - & 1 & - \\
\hline & Minéraux (mg..$\left.^{-1}\right)$ & 4,1 & - & 1 & 7,1 & - & 1 & - \\
\hline & Protéines (mg.g ${ }^{-1}$ poids sec) & 100,4 & - & 1 & 110,2 & - & 1 & - \\
\hline & Protéines $\left(\mathrm{mg} \cdot \mathrm{g}^{-1} \mathrm{MO}\right)$ & 110,5 & - & 1 & 120,8 & - & 1 & - \\
\hline & Énergie (kJ.g ${ }^{-1}$ poids sec) & 12,4 & - & 1 & 15,6 & - & 1 & - \\
\hline & Énergie (kJ.g-1 MO) & 15,7 & - & 1 & 18,5 & - & 1 & - \\
\hline & $\mathrm{P} / \mathrm{E}\left(\mathrm{mg} \cdot \mathrm{kJ}^{-1}\right)$ & 7,0 & - & 1 & 6,5 & - & 1 & - \\
\hline \multirow{9}{*}{ Sédiment } & Poids sec total $\left(\mathrm{g} \cdot \mathrm{m}^{-2}\right)$ & 1318,9 & 228,3 & 7 & 612,1 & 178,9 & 10 & 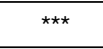 \\
\hline & $\mathrm{MOH}\left(\mathrm{g} \cdot \mathrm{m}^{-2}\right)$ & 225,8 & 52,1 & 7 & 178,7 & 70,3 & 10 & $\mathrm{~ns}$ \\
\hline & MORH $\left(\mathrm{g} \cdot \mathrm{m}^{-2}\right)$ & 98,1 & 23,5 & 7 & 45,4 & 19,3 & 10 & *** \\
\hline & Minéraux $\left(\mathrm{g} \cdot \mathrm{m}^{-2}\right)$ & 995,1 & 176,7 & 7 & 387,9 & 129,7 & 10 & *** \\
\hline & Protéines (mg.g ${ }^{-1}$ poids sec) & 72,3 & 24,3 & 7 & 90,5 & 31,6 & 10 & ns \\
\hline & Protéines $\left(\mathrm{mg} \mathrm{g}^{-1} \mathrm{MO}\right)$ & 98,5 & 39,4 & 7 & 106,2 & 47,8 & 10 & ns \\
\hline & Énergie (kJ.g ${ }^{-1}$ poids sec) & 9,7 & 5,3 & 7 & 10,1 & 6,6 & 10 & ns \\
\hline & Énergie (kJ.g $\left.{ }^{-1} \mathrm{MO}\right)$ & 13,9 & 10,7 & 7 & 18,3 & & 10 & ns \\
\hline & $\mathrm{P} / \mathrm{E}\left(\mathrm{mg} \cdot \mathrm{kJ}^{-1}\right)$ & 5,9 & 1,3 & 7 & 5,2 & 0,8 & 10 & ns \\
\hline \multirow{10}{*}{$\begin{array}{l}\text { Contenus } \\
\text { stomacaux }\end{array}$} & $\mathrm{MO}$ (mg. $\mathrm{g}^{-1}$ poids sec) & 453,8 & 99,5 & 9 & 536,1 & 143,5 & 10 & ns \\
\hline & $\mathrm{MOH}$ (mg.g ${ }^{-1}$ poids sec) & 362,7 & 98,4 & 9 & 443,7 & 141,6 & 10 & ns \\
\hline & MORH (mg.g ${ }^{-1}$ poids sec) & 91,1 & 7,6 & 9 & 92,4 & 11,8 & 10 & ns \\
\hline & Minéraux (mg.g ${ }^{-1}$ poids sec) & 546,2 & 99,5 & 9 & 463,9 & 143,5 & 10 & ns \\
\hline & Protéines (mg.g ${ }^{-1}$ poids sec) & 73,8 & 15,3 & 9 & 105,7 & 27,2 & 10 & ns \\
\hline & Protéines (mg.g $\left.{ }^{-1} \mathrm{MO}\right)$ & 94,8 & 11,0 & 9 & 141,3 & 19,3 & 10 & * \\
\hline & Lipides (mg.g ${ }^{-1}$ poids sec) & 110,9 & 48,4 & 9 & 70,7 & 30,5 & 10 & ns \\
\hline & Énergie (kJ.g ${ }^{-1}$ poids sec) & 12,2 & 2,2 & 9 & 9,4 & 1,6 & 10 & ns \\
\hline & Énergie (kJ.g-1 MO) & 15,7 & 3,3 & 9 & 11,3 & 8,7 & 10 & ns \\
\hline & $\mathrm{P} / \mathrm{E}\left(\mathrm{mg} \cdot \mathrm{kJ}^{-1}\right)$ & 6,0 & 4,7 & 9 & 12,5 & 3,8 & 10 & ** \\
\hline
\end{tabular}

$\mathrm{MOH}$ : Matière Organique Hydrolysable; MORH : Matière Organique Résistante à l'Hydrolyse; MO : Matière Organique totale ; P/E : rapport Protéines/Énergie. 
plus, la perturbation du milieu induite par l'activité de bioturbation des poissons fouisseurs comme Heterotis niloticus qui favorisent la remise en suspension le sédiment semble être importante. De ce fait, les teneurs en matières organiques hydrolysables des MES représentent environ $30 \%$ du poids sestonique avec, en corollaire, de faibles quantités de protéines apportées par cette composante (10 à $11 \%$ du poids sec total). En revanche,

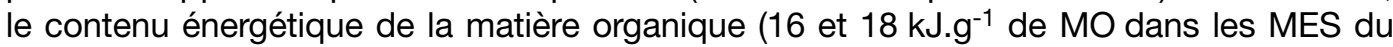
barrage 1 et 2, respectivement) est comparable aux valeurs caractéristiques du périphyton, des algues et des détritus benthiques (BOWEN, 1987). Le rapport P/E (7 mg.kJ-1 en moyenne) est environ 2,5 moins élevé que celui déterminé dans le périphyton.
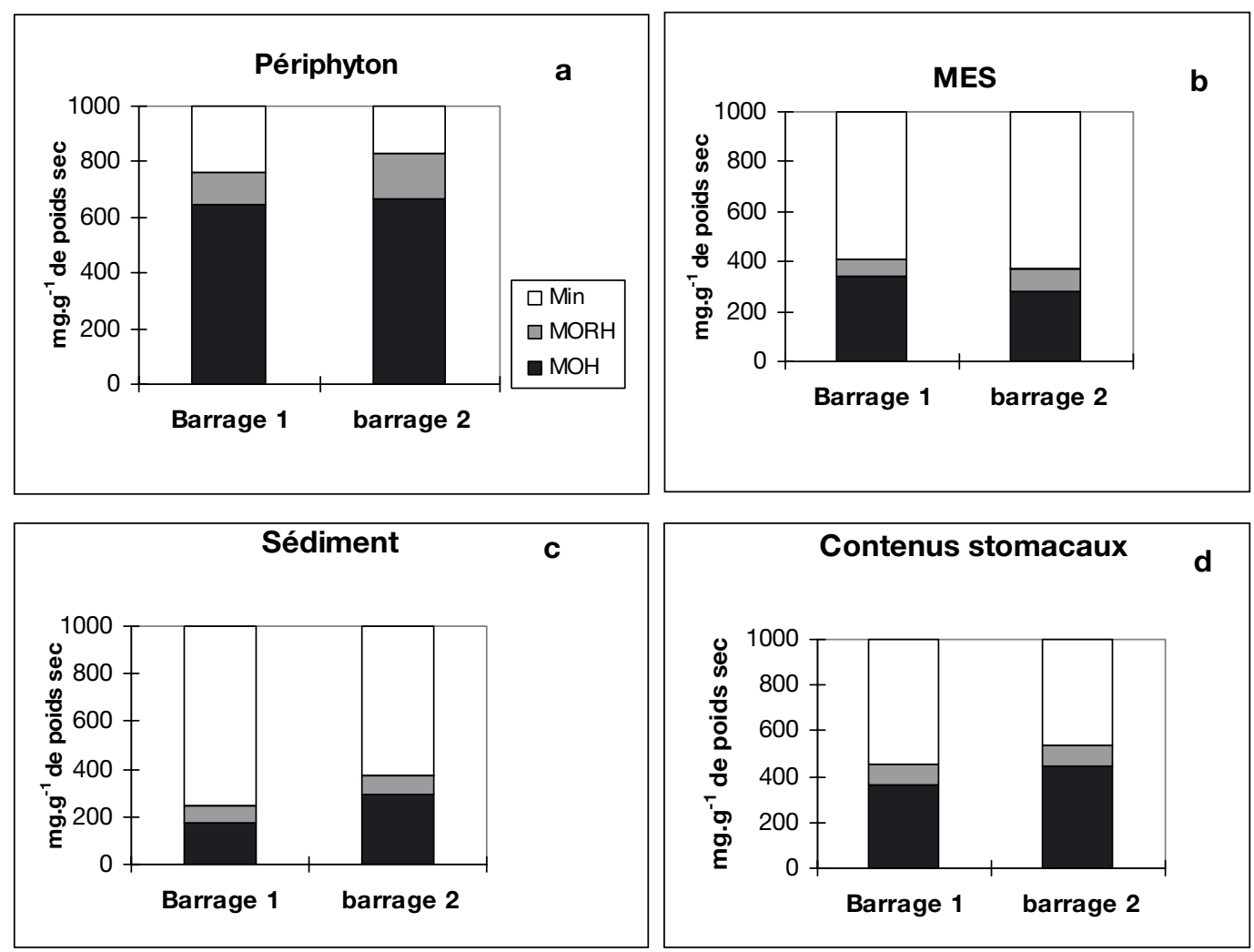

Min: Minéraux ; MORH : Matière organique résistante à l'hydrolyse ; $\mathrm{MOH}$ : Matière organique Hydrolysable

\section{Figure 1}

Composition chimique du périphyton (a), des MES (b), du sédiment (c) et des contenus stomacaux d'Oreochromis niloticus (d)

\section{Figure 1}

Chemical composition of periphyton (a), suspended solids (b), sediments (c) and gut contents of $O$. niloticus (d).

Les travaux relatifs aux caractéristiques chimiques et biochimiques des MES dans leur ensemble sont peu nombreux. La plupart des études traitent séparément du phytoplancton et des autres micro-organismes. Ainsi, les données bibliographiques sur les teneurs en protéines du phytoplancton, du zooplancton et des bactéries reflètent les potentialités nutritives de ces ressources, considérées comme étant d'une bonne qualité nutritionnelle pour les poissons microphages. Ainsi, le phytoplancton et le 
zooplancton peuvent présenter respectivement $27 \%$ et $60 \%$ de protéines (BOWEN, 1987). Dans certains étangs piscicoles ruraux du Centre-Ouest, les concentrations de matière organique du zooplancton et du phytoplancton atteignent respectivement $44 \%$ et $60 \%$ (DABBADIE, 1996). Ces valeurs, largement supérieures à celles observées dans les matières en suspension des deux barrages, semblent traduire une faible abondance de ces organismes au sein des MES des sites étudiés. En effet, les fortes proportions de minéraux, ajoutées à la faible transparence de l'eau $(0,4 \mathrm{~m}$ de profondeur de disparition du disque de Secchi) traduisent la forte turbidité de ces milieux. Ce facteur environnemental, souvent à la base de la disparition d'un grand nombre d'espèces phytoplanctoniques (SEVRIN-REYSSAC, 1995) contribuerait à expliquer les faibles teneurs en matière organique des MES. Par ailleurs, en absence d'apports exogènes d'aliments et de fertilisants pourvoyeurs de nutriments, les voies trophiques pélagiques sont peu stimulées pour favoriser un développement efficace des organismes planctoniques. Toutefois, la présence des poissons dans le milieu interfère sur les mesures du fait de leur action sélective sur les particules en suspension, ce qui contribue à sous-estimer la productivité réelle du compartiment pélagique.

La troisième source potentielle d'alimentation identifiée dans les barrages est constituée par la composante organique du sédiment superficiel. Les premiers centimètres de sédiment constituent un site important de dégradation et de stockage de la matière organique (BÉRARD, 1993). Les caractéristiques chimiques mesurées dans ce compartiment sont présentées à la Figure 1c. II apparaît également de fortes proportions de minéraux et de matières organiques résistantes à l'hydrolyse. Ces constituants non digestibles représentent respectivement 829 et $705 \mathrm{mg}^{-1} \mathrm{~g}^{-1}$ de poids sec de la ressource sédimentaire des barrages 1 et 2 . La fraction organique hydrolysable (digestible) représente moins de $30 \%$ de la ressource. Ramenées à des quantités par unité de surface, les teneurs en matières organiques hydrolysables $(\mathrm{MOH})$ sont comparables dans les deux sites (test non paramétrique de comparaison de moyennes, $p=0,143$ ) ; elles sont 12 fois plus élevées que celles fournies par le périphyton (Tableau I). Cela s'explique par l'importance des surfaces et des volumes concernés ; par ailleurs, l'épaisseur de la strate périphytique sur les substrats colonisés n'est pas la même que celle de la couche organique sédimentaire en raison de leurs caractéristiques distinctes. La couche de sédiment superficiel reçoit d'une part le flux du matériel qui sédimente et d'autre part, elle est enrichie par la matière organique d'origine détritique issue des morceaux de bois et des macrophytes en présence dans les barrages. A l'inverse, l'importance et la qualité de la biomasse du périphyton dépendent de la nature des substrats (AZIM et al., 2002a) et la productivité de sa composante algale est liée aux conditions environnementales du milieu (ARFI et al., 1997 ; BLÉ et al., 2006), notamment la turbidité qui caractérise souvent les barrages.

Au sein de la ressource sédimentaire, les matières organiques hydrolysables présentent une dispersion assez homogène au sein des barrages (CV $=25 \%$ et $23 \%$ dans les barrages 1 et 2, respectivement), avec toutefois un enrichissement plus prononcé en matériel organique au niveau des zones profondes situées en aval, près de la digue fermant l'ouvrage. Cette configuration est caractéristique des milieux lacustres où les particules minérales, plus lourdes, sédimentent en amont alors que la sédimentation plus en aval est essentiellement d'origine organique (CAMPY et MEYBECK, 1995). Ces caractéristiques nutritives du matériel sédimentaire présentent une certaine analogie avec celles des MES, en raison probablement des échanges et des flux de matières entre ce compartiment et la colonne d'eau. Ces caractéristiques se rapprochent également de celles étudiées dans le sédiment des acadjas en lagune Ébrié (BLE et al., 2006). Les quantités de protéines représentant moins de $10 \%$ du poids sec total de la ressource sont faibles par rapport à celles mesurées dans le matériel périphytique (Tableau I). Ces teneurs sont par contre comparables à celles déterminées dans les agrégats détritiques du lac Valencia (Venezuela) et dans les sédiments du lac Sibaya en Afrique du Sud (BOWEN, 1979 ; 1987). De plus, le contenu énergétique $\left(10 \mathrm{~kJ}^{-1}{ }^{-1}\right.$ de poids sec), ainsi que le ratio $\mathrm{P} / \mathrm{E}$ (autour 
de $6 \mathrm{mg} \cdot \mathrm{kJ}^{-1}$ ) s'intègrent dans l'intervalle de valeurs mesurées dans le compartiment détritique (BOWEN et al., 1995). Ceci reflète probablement l'origine détritique de la matière organique sédimentaire des barrages; cette hypothèse est soutenue par la présence de nombreux morceaux de bois et des macrophytes dans ces milieux, ajoutés aux apports allochtones (rejets d'origine anthropique, eaux de ruissellement). L'importance de la nourriture d'origine détritique dans le comportement alimentaire est fréquemment observée chez les poissons consommateurs primaires, particulièrement les herbivores et détritivores (LAUSANNE et ILTIS 1975 ; BOWEN, 1988). Dans les eaux résiduelles et les affluents du fleuve Niger, BAKARE (1970) note par exemple que Citharinus citharus sélectionne les détritus benthiques riches en matière organique. Bien que présentant des contenus protéiniques faibles et des rapports $P / E$ peu élevés, la source de nourriture que représentent les sédiments et les MES des barrages fournit une quantité importante de matières organiques hydrolysables pouvant être valorisée par les tilapias.

\section{Caractéristiques nutritives de la nourriture ingérée}

La composition chimique des contenus stomacaux des poissons est présentée à la Figure 1d. La matière organique totale ingérée représente respectivement 454 et $536 \mathrm{mg}$. $\mathrm{g}^{-1}$ du poids sec total des bols alimentaires issus des barrages 1 et 2 . La composante organique hydrolysable représentant la fraction digestible constitue 80 et $82 \%$ de ce matériel organique total. Les teneurs en matières organiques résistantes à l'hydrolyse restent relativement faibles, alors que les minéraux constituent une part importante, représentant respectivement 546 et $464 \mathrm{mg}^{-1} \mathrm{~g}^{-1}$ de la matière sèche ingérée. L'ensemble de ces composés non digestibles représente 64 et $56 \%$ des contenus stomacaux des poissons prélevés dans les barrages 1 et 2. Les proportions moyennes des constituants organiques et minéraux de ces bols alimentaires ne sont pas significativement différentes entre les deux sites, bien que l'alimentation des poissons du barrage 2 soit plus riche en matériel organique hydrolysable (Tableau I).

Cette composition chimique des contenus stomacaux est proche de celle des matières en suspension et du matériel sédimentaire, ce qui laisserait supposer que les poissons puisent majoritairement leur nourriture de ces deux sources alimentaires. Les barrages étant en effet soumis à un régime extensif, l'apport de composés nutritifs reste en grande partie dépendant de la nourriture naturelle et donc des compartiments trophiques les plus disponibles et accessibles, dont les MES et le sédiment. Les organismes périphytiques semblent être moins présents dans l'alimentation du fait probablement d'une biomasse insuffisante due à une moindre productivité, car ils doivent se développer dans un milieu turbide, ce qui limite l'épaisseur de la couche euphotique et donc du domaine dans lequel ils peuvent se développer. La qualité de la matière effectivement ingérée pourrait toutefois être sous-estimée par cette approche car l'alimentation des tilapias est non seulement sélective au niveau de chaque compartiment trophique (LAUSANNE et ILTIS, 1975 ; BOWEN, 1978 ; NORTHCOTT et al., 1991; GERKING, 1994) mais la composition du contenu stomacal peut être aussi modifiée par le début du processus digestif (MORIARTY, 1973).

A la différence des contenus en lipides et dans une moindre mesure des teneurs en fibres (assimilées dans notre étude aux matières organiques non hydrolysables) qui semblent répondre aux exigences nutritionnelles des tilapias (JAUNCEY et ROSS, 1982), les protéines sont présentes en faibles quantités, représentant respectivement 74 et $106 \mathrm{mg} \cdot \mathrm{g}^{-1}$ de poids sec dans les barrages 1 et 2 , soit 7 et $10 \%$ du poids sec des bols alimentaires des poissons (Figure 2). Ces teneurs traduisent une faible disponibilité en protéines de la nourriture ingérée, par comparaison aux besoins de ces poissons qui se situent entre 25 et $30 \%$ en fonction du contenu énergétique des régimes (SWEILUM et al., 2005 ; EL-SAIDY et GABER, 2005). Les rapports Protéines/Énergie mesurés dans les barrages ( 6 et $12 \mathrm{mg} \cdot \mathrm{kJ}^{-1}$ ), bien qu'étant supérieurs au minimum de 4 mg.kJ-1 requis dans l'alimentation chez les tilapias (BOWEN, 1982), sont également inférieurs à 


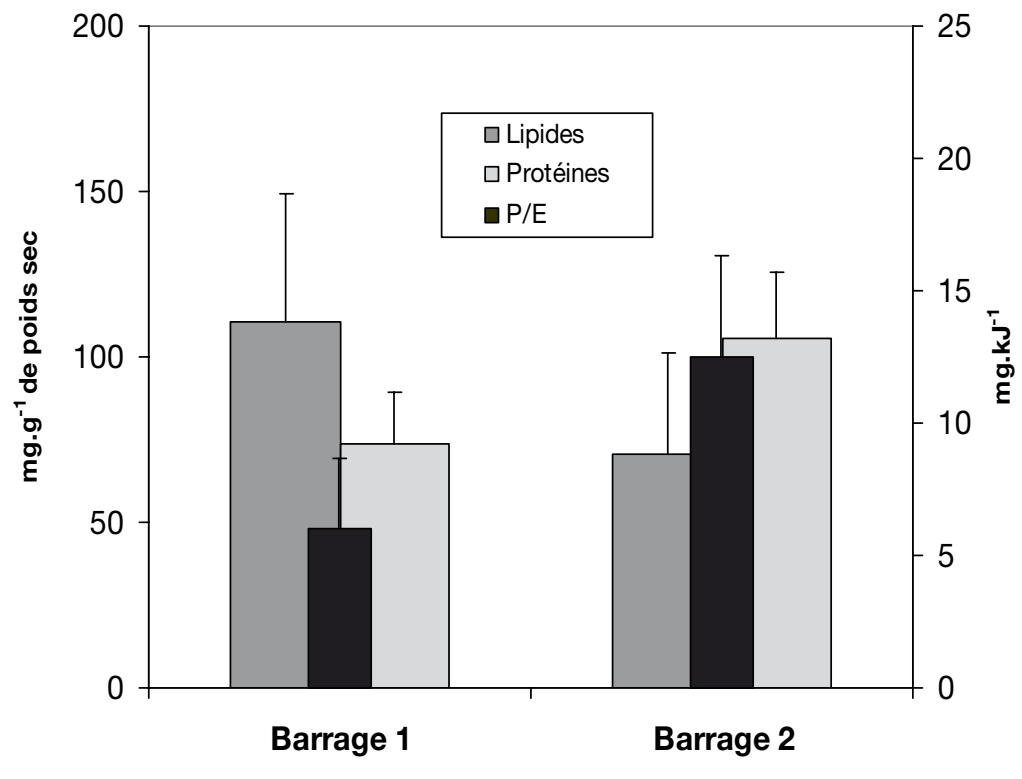

Lipides et Protéines exprimés en mg.g ${ }^{-1}$ de poids sec ; P/E en mg.kJ ${ }^{1}$

\section{Figure 2}

Teneurs moyennes en lipides et protéines et rapport Protéines/Energie P/E déterminés dans les contenus stomacaux d'O. niloticus.

\section{Figure 2}

Average concentration of lipids and proteins and ratio P/E measured in gut contents of 0 . niloticus.

\section{Tableau II}

Digestibilité moyenne de la matière organique hydrolysable (MOH) et des protéines de la nourriture ingérée par 0 . niloticus des deux barrages. Comparaison des moyennes à l'aide d'un test non paramétrique de Mann-Whitney.

\section{Table II}

Average digestibility of hydrolysable organic matter and proteins of food ingested by 0 . niloticus in the two reservoirs. Differences between groups are tested using parametric Mann-Whitney rank sum test.

\begin{tabular}{|c|c|c|c|c|c|c|c|}
\hline \multirow{2}{*}{ CUDa } & \multicolumn{3}{|c|}{ Barrage 1 } & \multicolumn{3}{c|}{ Barrage 2 } & \multirow{2}{*}{ Différences } \\
\cline { 2 - 7 } & Moyenne & $\begin{array}{c}\text { Écart- } \\
\text { type }\end{array}$ & $\mathbf{n}$ & Moyenne & $\begin{array}{c}\text { Écart- } \\
\text { type }\end{array}$ & $\mathbf{n}$ & \\
\hline $\mathrm{MOH}(\%)$ & 26,6 & 14,6 & 9 & 46,2 & 19,2 & 10 & $*$ \\
Protéines (\%) & 49,8 & 25,6 & 9 & 65,8 & 28,3 & 10 & ** \\
\hline
\end{tabular}

CUDa : Coefficient d'utilisation digestive apparente. 
la valeur optimale permettant une croissance efficiente chez 0 . niloticus. (18 mg.kJ ${ }^{-1}$, KAUSHIK et al., 1995). Ces caractéristiques nutritives présentent une similitude avec celles obtenues chez $O$. niloticus en milieu naturel, plus précisément dans certains lacs éthiopiens (GETACHEW, 1987 ; EYUALEM et GETACHEW, 1992). Dans le lac Chamo par exemple, la nourriture ingérée par ce poisson contient $45 \%$ de matière organique totale, $10 \%$ de matière organique résistante à l'hydrolyse et $10 \%$ de protéines (GETACHEW, 1993). Dans le lac Langeno, l'analyse des contenus stomacaux montre qu'ils sont généralement constitués de vase avec de faibles proportions en matières organiques (de l'ordre de $413 \mathrm{mg} \mathrm{g}^{-1}$ de poids sec). Les teneurs en protéines présentent cependant une forte variabilité saisonnière (TADESSE,1999).

La digestibilité de la matière organique et des protéines issues des ressources ingérées est présentée au Tableau II. Les valeurs concernant la matière organique sont faibles comparées aux valeurs de digestibilité obtenues expérimentalement à partir d'aliments naturels (BUDDINGTON, 1979 ; BOWEN, 1979 ; 1980 ; 1981). Par ailleurs, l'analyse des valeurs individuelles laisse apparaître d'importantes variations dans la digestibilité de la matière organique ou des protéines pour des rations identiques (CV $=55$ et $42 \%$ pour le CUDa de la matière organique et 51 et $43 \%$ pour le CUDa des protéines chez les poissons des barrages 1 et 2). Inversement, des contenus différents présentent souvent une digestibilité comparable pour ces deux nutriments. Les poissons disposant du même environnement trophique mais ayant probablement des moments d'alimentation différents, des phénomènes liés à la digestion plus ou moins avancée du contenu stomacal pourraient expliquer ces observations. Ces variations seraient également liées à l'évacuation gastrique différentielle des nutriments, en rapport avec la nature des aliments et le niveau de consommation (MORIARTY, 1973 ; GUILLAUME et CHOUBERT, 1999). Les techniques de collecte des fèces sont dans l'ensemble sujettes à discussion et sont souvent à la base des variations constatées dans les résultats de digestibilité chez les poissons (CHOUBERT, 1999). L'un des inconvénients de la méthode de dissection utilisée dans notre étude est qu'elle ne prendrait pas en compte l'ensemble des fèces émises au cours du processus digestif. De plus, une pollution éventuelle des fèces par l'enveloppe intestinale ou par une contamination sanguine pendant le prélèvement pourrait modifier leur composition et entraîner une mauvaise appréciation de la digestibilité (CHOUBERT, 1999 ; AMIRKOLAIE et al., 2005).

Globalement, la digestibilité des protéines est plus élevée (50 et $66 \%$ dans les barrages 1 et 2) par rapport au reste de la matière organique (27 et $46 \%$ ) et les résultats obtenus au cours de cette étude sont conformes à ceux observés dans les lacs éthiopiens pour la même espèce de poisson (EYUALEM et GETACHEW, 1992 ; TADESSE,1999). Les poissons du barrage 2 présentent une digestibilité des protéines plus élevée et cette différence semble être associée à la quantité des nutriments ingérés (Tableau I) : les teneurs moyennes de la fraction organique protéique et le rapport Protéines/Énergie des contenus stomacaux des poissons de ce barrage sont en effet plus élevés que dans le barrage 1. Un facteur explicatif probable est la densité d'empoissonnement qui est moins élevée dans le barrage 2, ce qui permet une disponibilité des ressources plus importante se traduisant par une meilleure digestion. Dans le cas du barrage 1, l'effet de la densité se traduirait par une dégradation de la qualité de la ration et cette observation semble être en accord avec la relation faite par BOWEN et al. (1995) qui montrent une baisse de digestibilité liée à une concurrence alimentaire en situation de carence en protéines de la ration, comme observé dans l'alimentation des poissons issus de ces barrages.

\section{Paramètres de croissance et de production}

Le Tableau III indique les résultats de croissance et de production en fin de cycle d'élevage dans les deux barrages. Les taux de survie (86 à $95 \%$ ) et les gains de masse moyenne obtenus montrent que ces milieux présentent un environnement plutôt favorable 
à l'élevage du tilapia O. niloticus. Par contre, dans les deux situations, la croissance reste relativement faible $\left(1 \mathrm{~g}_{\mathrm{j}}{ }^{-1}\right)$. Ce faible taux serait en partie lié à la composition chimique de la nourriture ingérée, caractérisée par une teneur élevée en matériel minéral qui pourrait provoquer une diminution de la sensation de réplétion chez les poissons et une baisse de leur croissance (MORIARTY et MORIARTY, 1973). Par ailleurs, le ratio Protéines/Énergie du bol alimentaire, variable affectant le taux d'ingestion et la croissance (BOWEN et al., 1995 ; SWEILUM et al., 2005 ; EL-SAIDY et GABER, 2005), parait faible pour soutenir correctement la croissance des poissons. Ce faible taux de croissance contraste cependant avec les biomasses moyennes (276 et $282 \mathrm{~g}$ dans les barrages 1 et 2) observées en fin de cycle. On peut donc suggérer en première approximation que la nourriture ingérée par ces poissons, bien que d'une qualité médiocre, fournit un contenu énergétique suffisant qui compléterait l'apport limité des sources protéiques pour soutenir les gains de poids. Cette hypothèse se rapprocherait des observations faites par KAUSHIK et al. (1995) et pourrait être assimilée aux adaptations physiologiques dont font preuve les poissons herbivores, leur permettant d'optimiser la qualité de leur nourriture en situation de faible disponibilité alimentaire (MELARD, 1986). Les fortes proportions de composés organiques hydrolysables représentant 80 à $82 \%$ de la matière organique totale ingérée, alors en faible quantité (45 à $54 \%$ ) dans les bols stomacaux, corroborent cette hypothèse et traduirait par la même occasion le caractère sélectif du comportement alimentaire du tilapia Oreochromis niloticus. Ces résultats sont également en relation avec les faibles densités d'empoissonnement appliquées et aux conditions du milieu, plus particulièrement les conditions d'oxygénation qui semblent être favorables à une utilisation efficiente de la nourriture disponible. Des observations similaires décrites en milieu naturel, tel le lac Chamo en Éthiopie (GETACHEW, 1993), confirment la moindre exigence trophique chez le tilapia Oreochromis niloticus et sa capacité à tirer profit des sources alimentaires de qualité variable.

\section{Tableau III}

Résultats du cycle de production.

\section{Table III}

Results of production cycle.

\begin{tabular}{lcc}
\hline \multicolumn{1}{c}{ Paramètres évalués } & Barrage 1 & Barrage 2 \\
\hline Poids moyen initial $(\mathrm{g})$ & $61,4 \pm 10,2$ & $13,6 \pm 6,8$ \\
Poids moyen final $(\mathrm{g})$ & $275,7 \pm 14,1$ & $282,5 \pm 98,7$ \\
Taux de survie (\%) & $95 \%$ & $86 \%$ \\
Gain moyen quotidien (g.j $\left.\mathrm{j}^{-1}\right)$ & 1,1 & 1,0 \\
Densité (ind. $\left.\mathrm{m}^{-2}\right)$ & 0,3 & 0,1 \\
Rendement $\left(\mathrm{t} . \mathrm{ha}^{-1} \cdot \mathrm{an}^{-1}\right)$ & 0,915 & 0,408 \\
\hline
\end{tabular}

\section{REMERCIEMENTS}

Ce travail a bénéficié de l'apport financier de l'Association Pisciculture et Développement Rural en Afrique Subsaharienne - Côte d'Ivoire (APDRACI). Les auteurs tiennent à remercier les Responsables de cette structure, particulièrement Monsieur Coulibaly Dramane, le Président de cette ONG ainsi que les correcteurs anonymes pour leurs remarques et suggestions. 


\section{BIBLIOGRAPHIE}

ALLEN G., 1981. Methods for detection of peptides. In: WORK T.S., BURDON R.H. (Eds.), Laboratory techniques in Biochemistry and Molecular, 135-160, North-Holland publications, New York.

ARFI R., BOUVY M., LUQUET P., 1997. Effect of seasonal salinity change on periphyton biomass in a shallow tropical lagoon. Int. Revue ges. Hydrobiol., 82, 81-93.

AMIRKOLAIE K.A., EL-SHAFAI A.S., EDING H.E., SCHRAMA W.J., VERRETH A.J., 2005. Comparison of faecal collection method with high- and low-quality diets regarding digestibility and faeces characteristics measurements in Nile tilapia. Aquaculture Research, 36, 578-585.

AZIM M.E., WAHAB M.A., VERDEGEM M.C.J., VAN DAM A.A., VAN ROOIJ J.M., BEVERIDGE M.C.M., 2002a. The effects of artificial substrates on freshwater pond productivity and water quality and the implications for periphyton-based aquaculture. Aquatic living resources, 15, 231-241.

AZIM M.E., VERDEGEM M.C.J., KHATOON H., WAHAB M.A., VAN DAM A.A., BEVERIDGE M.C.M., 2002b. A comparison of fertilization, feeding and three periphyton substrates for increasing fish production in freshwater pond aquaculture in Bangladesh. Aquaculture, 212, 227-243.

AZIM M.E., VERDEGEM M.C.J., MANTINGH I, VAN DAM A.A., BEVERIDGE M.C.M., 2003. Ingestion and utilization of periphyton growth on artificial substrates by Nile tilapia, Oreochromis niloticus L. Aquaculture Research, 34, 85-92.

BAKARE O., 1970. Bottom deposits as food of inland freshwater fish. In: VISSER S.A. (Eds.), Kainjin Lake Studies, University Press, Ibadan, Nigeria: 65 p.

BÉRARD A., 1993. Effets d'une fertilisation riche en matières organiques azotées sur les relations trophiques (bactéries, phytoplancton, zooplancton) dans un étang de pisciculture. Thèse doct., Muséum National d'Histoire Naturelle, Paris, France: $215 \mathrm{p}$.

BLE M.C., ARFI R., YEBOUA A.F., DIOPOH K.J., 2006. Qualité nutritive des sources alimentaires au sein d'un acadja (lagune Ébrié, Côte d'Ivoire, Afrique de l'ouest). Vie et Milieu, 56, 255-264.

BOWEN S.H., 1976. Mechanism for digestion of detrital bacteria by the cichlid fish Sarotherodon mossambicus (Peters). Nature, 260, 137-138.

BOWEN S.H., 1978. Benthic diatom distribution and grazing by Sarotherodon mossambicus in lake Sibaya, South Africa. Freshwater Biol., 8: 449-453.

BOWEN S.H., 1979. Determinants of the chemical composition of periphytic detrital aggregate in a tropical lake (Lake Valencia, Venezuela). Arch. Hydrobiol., 87, 166-177.

BOWEN S.H., 1980. Detrital non protein aminoacids are the key to rapid growth of tilapia in lake Valencia, Venezuela. Science, 207, 1216-1218.

BOWEN S.H., 1981. Digestion and assimilation of periphytic detrital aggregate by Tilapia mossambica. Transactions of the American Fisheries Society, 110, 239-245.

BOWEN S.H., 1982. Feeding digestion and growth - Qualitative consideration. In: PULLIN R.S.V., LOWE Mc CONNELL R.H. (Eds.), The biology and culture of tilapias, 141156, ICLARM conf. proceedings, 7, Manila, Philippines.

BOWEN S.H., 1983. Quantitative description of diet. In: NIELSON L.A., JOHNSON D.L. (Eds.), Fisheries Techniques, 325-336, American Fisheries Society, Bethesda, Maryland. 
BOWEN S.H., 1987. Composition and nutritional value of detritus. In: MORIARTY D.J.W., PULLIN R.S.V. (Eds.), Detritus and microbial ecology in aquaculture, 192-216, ICLARM conference proceedings, 14, Manila, Philippines.

BOWEN S.H., 1988. Detritivory and herbivory. In : LEVÊQUE C., BRUTON M.N., SSENTONGO G.W. (Eds.), Biologie et écologie des poisons d'eau douce africains, 243-247, Collection Travaux et Documents, 219, ORSTOM, Paris, France.

BOWEN S.H., LUTZ E.V., AHLGREN M.O., 1995. Dietary protein and energy as determinants of food quality: trophic strategies compared. Ecology, 76, 899-907.

BUDDINGTON R.K., 1979. Digestion of an aquatic macrophyte by Tilapia zilli (Gervais). Journal of Fish Biology, 15, 449-455.

BUDDINGTON R.K., 1980. Hydrolysis-resistant organic matter as a reference for measurement of fish digestive efficiency. Transactions of the American Fisheries Society, 109, 653-655.

CAMPY C., MEYBECK M., 1995. Les sédiments lacustres. In POURRIOT R., MEYBECK M. (Eds.), Limnologie générale, 185-226, Coll. Écol., 25, Masson, Paris.

CHOUBERT G., 1999. La digestibilité des nutriments chez les poisons: Aspects de méthodologie. Cybium, 23, 113-125.

CONOVER R.J., 1966. Assimilation of organic matter by zooplankton. Limnol. Oceanogr., $11,338-345$.

DABBADIE L., 1996. Étude de la viabilité d'une pisciculture rurale à faible niveau d'intrant dans le Centre-Ouest de la Côte d'Ivoire: Approche du réseau trophique. Thèse de doctorat, Université Paris VI, France : 208 p.

DEMPSTER P.W., BEVERIDGE M.C.M., BAIRD D.J., 1993. Herbivory in the tilapia Oreochromis niloticus: a comparison of feeding rates on phytoplankton and periphyton. Journal of Fish Biology, 43, 385-392.

EL-SAIDY D.M.S.D., GABER M.M.A., 2005. Effect of protein levels and feeding rates on growth performance, production traits and body composition of Nile tilapia, Oreochromis niloticus (L.) cultured in concrete tanks. Aquaculture research, 36, 163-171.

EYUALEM A., GETACHEW T., 1992. Seasonal changes in the nutritional status of Oreochromis niloticus LINN (Pisces: Cichlidae) in lake Ziway, Ethiopia. Arch. Hydrobiol., 124, 109-122.

GETACHEW T., 1987. A study on an herbivorous fish, Oreochromis niloticus L., diet and its quality in two Ethiopian Rift Valley lakes, Awasa and Zwai. Journal of Fish Biology, 30, 439-449.

GETACHEW T., 1993. The composition and nutritional status of diet of Oreochromis niloticus in Lake Chamo, Ethiopia. Journal of Fish Biology, 42, 865-874.

GERKING S.D., 1994. Feeding ecology of fish. Academic Press, San Diego, États-Unis: $416 \mathrm{p}$.

GOLTERMAN H. L., CLYMO R.S., OHSTAND M.A.M., 1978. Methods for physical and chemical analysis of freshwater. $2^{\text {nd }}$ ed. IBP Handbook No. 8. Blackwell Scientific Publications, Oxford. 1-213.

GUILLAUME J., CHOUBERT G., 1999. Physiologie digestive et digestibilité des nutriments chez les poissons. In : GUILLAUME J., KAUSHIK S., BERGOT P., METAILLER R. (Eds.), Nutrition et alimentation des poisons et crustacés, 51-86, INRA éditions. 
GUIRAL D., ARFI R., KOUETÉ P.D., KONAN-BROU A.A., 1993. Communautés, biomasses et productions algales au sein d'un récif artificiel (acadja) en milieu lagunaire tropical. Rev. Hydrobiol. Trop. 26, 219-228.

HUCHETTE M.S.H., BEVERIDGE M.C.M., BAIRD D.J., IRLAND M., 2000. The impacts of grazing by tilapias (Oreochromis niloticus L.) on periphyton communities growing on artificial substrate in cages. Aquaculture, 186, 45-60.

JAUNCEY K., ROSS B., 1982. A guide to tilapia feeds and feeding. Institute of aquaculture, University of Stirling, Scotland, $111 \mathrm{p}$.

KAUSHIK S.J., DOUDET T., MEDALE F., AGUIRRE P., BLANC D., 1995. Protein and energy needs for maintenance and growth of Nile tilapia (Oreochromis niloticus). J. Appl. Ichthyol., 11, 290-296.

KESHAVANATH P., GANGADHAR B., RAMES T.J., VAN ROOIJ J.M., BEVERIDGE M.C.M., VERDEGEM M.C.J., VAN DAM A.A., 2001. Use of artificial substrates to enhance production of freshwater herbivorous fish in pond culture. Aquaculture Research, 32, 189-197.

LAUZANNE L., 1978. Étude quantitative de l'alimentation de Sarotherodon galilaeus (Pisces, Cichlidae) du lac Tchad. Cah. ORSTOM, série. Hydrobiol., 12, 71-81.

MELARD C., 1986. Les bases biologiques de l'élevage intensif du tilapia du Nil. Cahiers d'étiologie appliquée, 6, 224 p.

MORIARTY D.J.W., 1973. The physiology of digestion of blue-green algae in the cichlid fish Tilapia nilotica. J. Zool., Lond., 171, 25-39.

MORIARTY C.M., MORIARTY D.J.W., 1973. Quantitative estimation of the daily ingestion of phytoplankton by Tilapia nilotica and Haplochromis nigripinnis in lake George, Uganda. J. Zool., Lond., 171, 15-23.

MORISSENS P., OSWALD M., SANCHEZ F., HEM S., 1996. Approche des nouveaux modèles d'exploitation piscicole adaptés au contexte rural ivoirien. In : PULLIN R.S.V., LAZARD J., LEGENDRE M., AMON KOTHIAS J.B., PAULY D., (Eds), Le troisième symposium International sur le tilapia en aquaculture, 130-141. ICLARM conf. Proc. 41 p.

NORTHCOTT M.E, BEVERIDGE M.C.M, ROSS L.G., 1991. A Laboratory investigation of the filtration and ingestion rates of the tilapia, Oreochromis niloticus, feeding on two species of blue-green algae. Environ. Biol. Fishes, 31, 75-85.

SCHROEDER G.L., 1978. Autotrophic and heterotrophic production of micro-organisms in intensively-manured fish ponds and related fish yields. Aquaculture, 14, 303-325.

SEVRIN-REYSSAC J., 1995. Le réseau alimentaire dans les étangs de cypriniculture. In : BILLARD R., (Éd.), Les carpes, biologie et élevage, 25-55. INRA, Paris, France.

SWEILUM M.A., ABDELLA M.M., EL-DIN S.A.S., 2005. Effect of dietary protein-energy levels and fish initial sizes on growth rate, development and production of Nile tilapia, Oreochromis niloticus L. Aquaculture Research, 36, 1414-1421.

TADESSE Z., 1999. The nutritional status and digestibility of Oreochromis niloticus L. diet in Lake Langeno, Ethiopia. Hydrobiologia, 416, 97-106.

WINDELL T.W., BOWEN, S.H., 1978. Methods for Study of Fish Diets Based on Analysis of Stomach Contents. In: BAGENA T. (Ed.), Methods for Assessment of fish production in freshwaters, 219-226, $3^{\text {rd }}$. Édition. Blackwell scientific publication, Oxford, IBP handbook. 3. 